\title{
EFFECTS OF CP-154,526 AND VERAPAMIL ON CORTICOTROPHIN RELEASING FACTOR SECRETING LEVELS
}

\author{
J. Dong ${ }^{1,2}$, Y.M. Zhu ${ }^{1}$, W.W. Zhou ${ }^{3}$, F. Yuan ${ }^{1}$, C. Zhang ${ }^{1}$, C.Y. Dong ${ }^{1}$, Z.H. Xiao ${ }^{1}$, Y.H. Gui ${ }^{2}$, C. Chen ${ }^{2}$ \\ ${ }^{I}$ Pediatric Intensive Care Unit, Children's Hospital of Hunan Province, Changsha City, ${ }^{2}$ Children's Hospital \\ of Fudan University, Shanghai, ${ }^{3}$ Department of Chest Surgery, Children's Hospital of Hunan Province, \\ Changsha City, China
}

Background and aims: We discovered that plasma corticotrophin releasing factor (CRF) levels in the young rats with hypoxic-ischemia (HI) brain damage were changeable, which was related with the time of HI. In present study, we will explore the effects of CRF acceptor 1 (CRF-R1) antagonist CP-154,526 (CP) and $\mathrm{Ca}^{2+}$ ion pathway antagonist Verapamil (VP) on plasma CRF secreting levels in the young rats after hypoxicischemia for one day.

Methods: Eighty young rats were randomly divided into eight groups, the normal control groups, sham operated groups, CP control groups, VP control groups, model groups, $\mathrm{HI}+\mathrm{CP}$ groups, $\mathrm{HI}+\mathrm{VP}$ groups and $\mathrm{HI}+\mathrm{CP}+\mathrm{VP}$ groups are set. Plasma CRF levels of young rats in all groups were measured by radioimmunoassay.

Results: Compared with the normal control groups, sham operated groups, CP control groups, VP control groups, plasma CRF levels in the model groups, $\mathrm{HI}+\mathrm{CP}$ groups, $\mathrm{HI}+\mathrm{VP}$ groups and $\mathrm{HI}+\mathrm{CP}+\mathrm{VP}$ groups all significantly decreased (P Values all than less 0.001). Compared with the HI groups, plasma CRF levels in the $\mathrm{HI}+\mathrm{CP}$ groups $(\mathrm{P}<0.05)$ and $\mathrm{HI}+\mathrm{VP}$ groups $(\mathrm{P}<0.001)$ all significantly increased. Compared with the $\mathrm{HI}+\mathrm{CP}$ groups, plasma CRF levels in the $\mathrm{HI}+\mathrm{VP}$ groups $(\mathrm{P}<0.001)$ significantly increased. Compared with the $\mathrm{HI}+\mathrm{VP}$ groups, plasma $\mathrm{CRF}$ levels in the $\mathrm{HI}+\mathrm{CP}+\mathrm{VP}$ groups $(\mathrm{P}<0.001)$ significantly decreased.

Conclusions: CRF-R1 antagonist $\mathrm{CP}$ or $\mathrm{Ca}^{2+}$ ion pathway antagonist VP can promote CRF secreting levels in the young rats after hypoxic-ischemia for one day, but when the CP and VP were simultaneously used, and $\mathrm{CP}$ can deteriorate the CRF secreting levels. 